\title{
Modelling the mobility of positive ion clusters in normal liquid helium over large pressure ranges
}

\author{
Frédéric Aitken, ${ }^{* a}$ Nelly Bonifaci, ${ }^{a}$, Luis Guillermo Mendoza-Luna, ${ }^{b}$ and Klaus von Haeften ${ }^{b}$
}

Received Xth $X X X X X X X X X X 20 X X$, Accepted Xth $X X X X X X X X X 20 X X$

First published on the web Xth $X X X X X X X X X X 200 X$

DOI: 10.1039/b000000x

Positively charged helium clusters, also called 'snowballs', have been investigated within normal liquid helium. Thermodynamic state equations for ionic helium clusters in liquid helium have been developed, allowing us to discern the 'hydrodynamic' radius for a wide range of hydrostatic pressures and temperatures. The mobilities derived from the cluster sizes using Stokes law match experimental data with unsurpassed accuracy. For low pressures the compressibility of the cluster ions was found to be distinctly larger than the compressibility of solid helium suggesting that in this pressure range clusters are fully or partially liquid.

\section{Introduction}

The investigation of ions in liquids is of considerable interest for the understanding of interactions in bulk liquids ${ }^{1,2}$ as well as for applications, for examples ionic liquids ${ }^{3-5}$ or chemical analysis 6,7 . Attractive interaction between ions and the solvent liquid gives rise to the formation of clusters whose size can be elucidated by measuring their mobility. The modelling of ion mobility is nevertheless a great challenge since fluid properties have to be taken into account ${ }^{8,9}$.

Liquid helium has played a special role in this context because it can serve in many respects as a model. Compared to other solvents it is practically free of foreign impurities. Furthermore, it is non-polar, consists of atoms with few electrons and therefore appealing for theory. In addition, liquid helium exhibits quantum effects which can be explored through solute-solvent interaction at the nanoscale.

In view of the high purity of liquid helium and its practicality, helium ions are among the first choices as solutes. Ionisation of liquid helium results in the formation of positively charged helium clusters, commonly referred to as 'snowballs', whose formation is driven by the balance of electrostrictive attraction ${ }^{10-12}$ and short-range repulsive exchange forces ${ }^{13}$. The existence of snowballs became first evident in mobility measurements about 50 years ago when very low mobilities associated with unusually large hydrodynamic radii of the charged particles were discovered ${ }^{12}$. Atkins calculated the size of a snowball by depicting the liquid helium as a classical dielectric continuum and by postulating a surface energy equivalent to the surface tension of liquid helium ${ }^{11}$. The high

${ }^{a}$ G2ELab-CNRS, 25 Av. des Martyrs BP 166, 38042 Grenoble, Cedex 9, France; E-mail: frederic.aitken@grenoble.cnrs.fr

${ }^{b}$ Department of Physics and Astronomy, University of Leicester, University Road, Leicester, LE1 7RH, United Kingdom pressure arising from electrostriction suggested a solid structure in the immediate vicinity of the positive ion in the centre of the cluster. Mixed quantum/classical dynamics calculations and, more recently, density functional theory studies identified the linear triatomic $\mathrm{He}_{3}^{+}$ion as the centre of an isolated snowball cluster ${ }^{14,15}$, which should have an effect on the mobility ${ }^{16}$. The picture of a solid snowball forming around a positive core was also found valid for foreign ions and has received both experimental and theoretical support: on the experimental side the long life-time of nuclear spin polarised ${ }^{12} \mathrm{~B}^{+}$and ${ }^{8} \mathrm{Li}^{+}$ions injected into liquid helium ${ }^{17}$ has been attributed to helium snowballs forming around the positively charged foreign ion; the observation of magic numbers in the time-of-flight mass spectrum of $\mathrm{He}_{n} \mathrm{Cs}^{+}$and $\mathrm{He}_{n} \mathrm{Ar}^{+}$clusters ejected from large doped helium droplets ${ }^{18,19}$ showed the prevalence of specific - hence solid - structures; on the theoretical side, Diffusion Monte Carlo (DMC) calculations for small ${ }^{4} \mathrm{He}$ clusters containing alkali ions revealed solid-like snowballs surrounded by a liquid-like environment of additional solvent atoms ${ }^{20-22}$. Also radial density profiles from DMC calculations for $\mathrm{Pb}^{2+} \mathrm{He}_{n}$ for several relevant sizes $n$ show peak helium density values much higher than the density of solid helium ${ }^{23}$. An investigation of the photoionisation of $\mathrm{Rb}$ and $\mathrm{Cs}$ residing at the surface of helium droplets using density functional theory showed that both solvated and desorbed ions form snowball structures ${ }^{24}$.

Helium snowballs are relevant to several scientific domains: they constitute probes for exploring the normal liquid and superfluid phase of helium ${ }^{10,12,25}$, they are seeds for the solidification of superfluid helium ${ }^{26}$, they are observed as reaction products in gas phase ionisation experiments using helium droplet beams ${ }^{18,27-29}$ and they are model systems for ion-molecule interaction ${ }^{30}$ and solvation chemistry. Recently, it was pointed out that snowballs in liquid helium might be dy- 
namical objects that on the one hand have a well-defined mass, but which on the other hand allow exchange of solute and solvent helium atoms ${ }^{23}$. Studying helium ion clusters might ultimately add to a better understanding of the solvation of ions in polar solvents of more practically relevant systems.

In view of the relevance of helium snowballs in various fields the availability of accurate size data is imperative. Despite the popularity of the Atkins model, its ability to describe the mobility of positive helium ions in liquid helium is limited $^{31}$, particularly at low and high hydrostatic pressures ${ }^{32}$. This is illustrated in Fig. 1 which shows measured and simulated mobilities using the Atkins model for liquid helium in a wide range of pressures from $0.1 \mathrm{MPa}$ to $6 \mathrm{MPa}$ for isotherms at 2.6, 3.0 and $4.2 \mathrm{~K}$. Since the surface tension of helium at the snowball-liquid interface is unknown the calculation was performed for two limiting cases using $\sigma=0 \mathrm{mN} / \mathrm{m}$ and $\sigma=0.1 \mathrm{mN} / \mathrm{m}$ as surface tension coefficients. Significant deviations are observed. Irrespective of the choice of the parameters the calculated mobilities shown in Fig. 1 represent the best fit over a large pressure range one can obtain.

Several reasons for the observed deviations can be identified: for the fluid Atkins assumed a radially decreasing density, produced by the radially decaying electrostrictive forces. All other relevant quantities such as viscosity were derived from these densities and consequently showed the same radial dependence. A consequence of the assumption of a radial dependence of the fluid properties is that the drag force is no longer given by Stokes law and a new solution of the NavierStokes equations has to be found. Apart from this conceptual problem, the mobilities depend on the surface tension which is only defined at the gas-liquid coexistence curve.

The present paper addresses the issue of accurate prediction of the mobility of positive ions in liquid helium and its hydrostatic pressure dependence, taking fluid properties fully into account. Previously published ion mobility data is revisited and compared with calculated mobility using a thermodynamic approach and the free volume model. Earlier work on electrons in liquid, supercritical and gaseous helium adopting this approach has produced excellent agreement with experimental data, reproducing the variation of mobility with pressure for the first time correctly ${ }^{36,37}$. The method rests on state equations for the mixed system of a small concentration of snowballs in liquid helium. In doing so, the entire fluid properties over a wide range of temperatures and pressures are taken into account, whereas unknowns in the Atkins model such as structure of the snowball, properties of the liquid-solid interface and of the surrounding liquid are bypassed. The freevolume model is then employed to derive the hydrodynamic radius and the mobility via Stokes law. While the form of the state equation has been chosen to reproduce experimental data most accurately, the equations contain parameters which are calibrated using experimental mobility data on one randomly

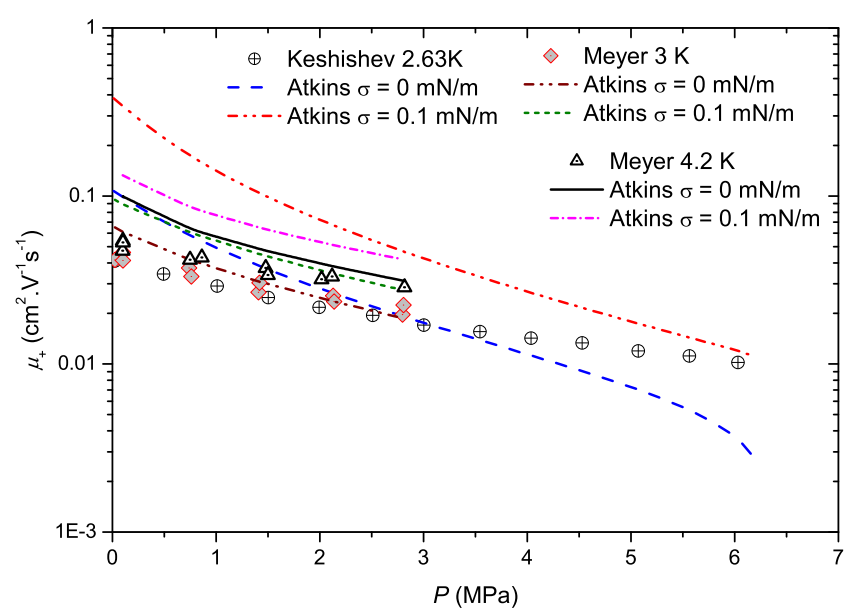

Fig. 1 Experimentally derived mobilities of positive ions in liquid helium (symbols) at $2.63 \mathrm{~K}^{33}, 3 \mathrm{~K}^{34}$ and $4.2 \mathrm{~K}^{34}$. The first data point of each graph lies on the saturated vapour-pressure (SVP) curve and in all cases it has been taken from Schwarz ${ }^{35}$. The dashed dotted lines show mobilities calculated using the Atkins model for the respective experimental conditions. For each temperature two calculations were performed using $\sigma=0 \mathrm{mN} / \mathrm{m}$ and $\sigma=0.1 \mathrm{mN} / \mathrm{m}$ as surface tension coefficient. The calculated mobilities deviate at low and high pressures irrespective of the chosen surface tension coefficient. The variation with pressure deviates consistently from the experimental data.

chosen isotherm as benchmark. The calculated mobilities on all other isotherms are found to match experimental data very well - much better than the Atkins model.

Furthermore, the simulated pressure dependence of the hydrodynamic radius in the normal liquid phase of helium is employed to calculate the isothermal compressibility, assuming spherically shaped clusters. The calculated compressibilities are smaller than those of pure liquid helium at similar temperature but larger than the reported values of the solid phase of pure helium, indicating that the solid structure of the snowballs is not fully developed across the clusters.

\section{Method}

\subsection{Modelling mobility and hydrodynamic radius}

2.1.1 The free volume model. The free volume model is used to derive the size of an unknown foreign microscopic object that coexists in thermal equilibrium at low concentration. In the past, the free volume concept has been applied successfully to model transport properties of fluids ${ }^{38-40}$. Gee and Freeman have used it to calculate the mobility of electrons and ions in alkanes and in carbon disulphide ${ }^{41,42}$. In the present paper Stokes law is used to relate the mobility to a size. The size of an ion is then modelled separately from the 
viscosity.

A key assumption is the proportionality of the volume occupied by an unknown foreign object and the volume $(V-b) / N$ available to single helium atoms. Here, $V$ designates the total volume of an ensemble of $N$ helium atoms and $b$ the covolume of the helium. This proportionality means that the volume of the foreign object changes in the same fashion as the volume, $V$, that is available to the helium. Strictly speaking, the co-volume, $b$, of all hard-sphere helium atoms in the ensemble has to be replaced by a different co-volume $b^{\prime}$ because the foreign particles contribute to the co-volume with a different hard sphere volume, however this contribution is negligibly small. The free volume model introduces a new quantity, the free volume $V_{f}$ which is equal to the difference $V-b^{\prime}$ and for which the state equation is formulated. We write for the free volume $V_{f}=V-b^{\prime}$ and for the volume of a snowball $V_{\text {ion }}$

$$
V_{\text {ion }}=\operatorname{const} \frac{V_{f}}{N}
$$

where const is the proportionality constant.

For small concentrations of foreign particles the total volume of the system will not change much and we can relate the volume occupied by the impurities to $P$ and $T$ thereby establishing a state equation for positive ions in normal liquid helium.

\subsubsection{Development of thermodynamic state functions.}

A thermodynamic state function relates $P, V$, and $T$ such that for a given pair of the three variables the third can be calculated. Any equation that reproduces experimentally determined relations between $P, V$, and $T$ is valid. In our approach, the reference for the validity of the thermodynamic state equations for positive ions in liquid helium is the experimentally determined mobility. An important conclusion from this approach is that all thermodynamic state equations that fulfil this requirement have to be valid because they show the correct variation of volume or density, respectively, with pressure and temperature.

For the development of state equations we were guided by the experimentally determined mobility of positive ions in normal liquid helium. Inspection of the variation of the mobility with pressure and temperature suggested a van der Waals-type relation between free volume, pressure and temperature as follows.

$$
V_{f}=\frac{N k_{B} T}{P+\Pi}
$$

Here $k_{B}$ is the Boltzmann constant, and $\Pi$ is the internal pressure that accounts for all attractive interactions, i.e. between the impurities and the pure helium atoms.

If we assume the region occupied by the foreign object to be spherical and of volume $V_{\text {ion }}=(4 / 3) \pi r^{3}$ we can express the effective snowball radius, $r$, by

$$
r=C_{i o n} \sqrt[3]{\frac{3}{4 \pi} \frac{k_{B} T}{P+\Pi}}
$$

It is useful to consider a variable, $r$, and an invariable part, $a$, of $r=r^{\prime}-a$ to account for the hard-sphere radius of the unsolvated helium ion. For the hard-sphere radius we find a good fit for $a=0.74 \AA$, a result that is further supported from an investigation of positive ion mobility in supercritical helium which will be presented in a forthcoming publication.

Consequently $r^{\prime}-a$ represents the snowball radius in excess of the radius of the 'naked ion'. For convenience, we write $C_{i o n}$ instead of $\sqrt[3]{1 / \text { const }}$ where the index $a$ will change if another foreign particle with a different interaction is considered.

Finding the snowball radius $r$ reduces to the problem of determining $C_{i o n}$ and $\Pi$, which is achieved by calibration of the parameters of the state equations to the hydrodynamic radius derived from experimentally determined mobility and by developing an expression for the internal pressure $\Pi$ in Eq. (3) that shows consistent variation of the mobility with $P, T$ and $\rho$. For positive ions in normal liquid helium $C_{i o n}$ is found to be largely independent of the temperature and the calibration of $C_{i o n}$ has to be performed only once, on one isotherm. For calibration, we used the data from Meyer ${ }^{34}$ and found $C_{i o n}=5.102$. This value agrees within $20 \%$ with the mobility reported by Schwarz ${ }^{35}$ and Kuchnir ${ }^{43}$ for the liquid coexistence line (saturated vapour pressure line) which is also within the sum of the errors reported by both authors.

After $C_{i o n}$ and the parameters in $\Pi$ have been determined, Eq. (3) can be used for calculating the radii, $r$. Since the dynamic viscosity of helium, $\eta$, is well documented for a wide pressure range and temperatures in normal liquid helium ${ }^{44}$, it is possible to calculate the mobility via the Stokes-Einstein equation (4)

$$
\mu_{\text {Stokes }}=\frac{e}{6 \pi r \eta}
$$

2.1.3 Internal pressure $\Pi$ and hydrodynamic radius $r$ of positive ions in liquid helium. All equations describing stable thermodynamic systems must have a first-order proportionality of $\Pi$ with $\rho^{2}$. Higher-order corrections are used to account for specific interactions of the system. For the normal liquid phase of helium we find best match with experimental data for an expression for $\Pi$ that is directly proportional to $\alpha \rho^{2}$ over a very large range of pressures and temperatures. Only for high pressures close to the solidification line, and for temperatures close to the $\lambda$-line higher-order corrections are 


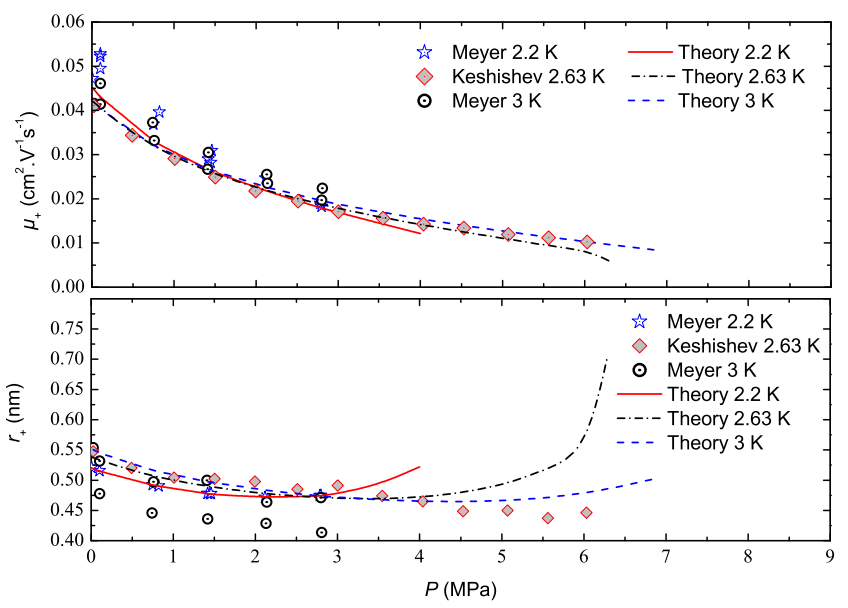

Fig. 2 Mobility and hydrodynamic radius of positive ions in the low temperature region of normal liquid helium at $2.2 \mathrm{~K}, 2.63 \mathrm{~K}$ and 3.0 K. The experimental data has been taken from Meyer et al. ${ }^{34}$ and Keshishev ${ }^{33}$. The first point of each graph lies on the saturated vapour pressure curve and is always from Schwarz ${ }^{35}$. Notice that the deviations of the theoretical curve from the experimental data are of the order of $0.1 \mathrm{~nm}$.

needed.

$$
\begin{aligned}
& \Pi(\rho, T)= \\
& \begin{cases}\alpha \rho^{2}\left[1-\left(\frac{\rho-\rho_{\text {sat }}}{\rho_{\text {sol }}-\rho_{\text {sat }}}\right)^{\left.\frac{T}{T_{\lambda, \max }} \varphi\left(\frac{\rho}{\rho_{\lambda}}\right)\right]}\right. & \text { if } \rho \leq \rho_{\text {sol }} \\
0 & \text { if } \rho>\rho_{\text {sol }}\end{cases}
\end{aligned}
$$

with

$$
\varphi\left(\frac{\rho}{\rho_{\lambda}}\right)=5-\frac{4}{1+\exp \left(\frac{\frac{\rho}{\rho_{\lambda}}-1}{\Delta}\right)},
$$

$\alpha=0.00796 \mathrm{bar} \mathrm{m}^{6} / \mathrm{kg}^{2}$ and $\Delta=0.00317 . \rho_{\text {sat }}, \rho_{\text {sol }}$ and $\rho_{\lambda}$ are functions of the temperature and represent the densities at saturated vapour pressure, solid helium and the $\lambda$-line, respectively. $\rho$ has to be entered in $\mathrm{kg} / \mathrm{m}^{3}$.

The correction with respect to strict proportionality to $\alpha \rho^{2}$ is dominated by $\varphi$ as per Eq. (6). A comparison of $\Pi$ with and without correction term is shown elsewhere ${ }^{45}$.

\section{Results and discussion}

Figs. (2) and (3) show the pressure dependence of mobility, $\mu$, and radius, $r$, for normal liquid helium at temperatures of $2.2 \mathrm{~K}, 2.63 \mathrm{~K}, 3.0 \mathrm{~K}, 4.2 \mathrm{~K}$ as measured by Meyer ${ }^{34}$ and Keshishev ${ }^{33}$ For all temperatures the mobilities decrease with increasing pressure.

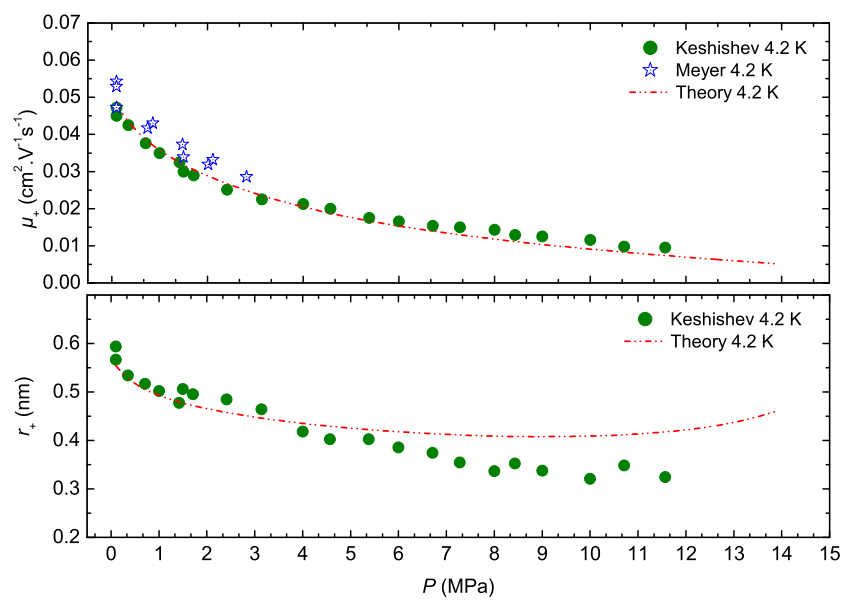

Fig. 3 Mobility (upper panel) and hydrodynamic radius (lower panel) of positive ions in the high temperature region of normal liquid helium at $4.2 \mathrm{~K}$. The experimental data has been taken from Meyer et al. ${ }^{34}$ and Keshishev ${ }^{33}$. The first point of each graph lies on the saturated vapour pressure curve and is always from Schwarz ${ }^{35}$.

The radii shown were deduced from the measured mobilities using Eq. (4) and compared with calculated radii using Eqs. (3) and (5). The radii decrease as well, up to a turnaround which for temperatures at $2.2 \mathrm{~K}$ lies at pressures of about $2.5 \mathrm{MPa}$, which is close to the melting line of solid helium. For temperatures at $4.2 \mathrm{~K}$ this turnaround is more shallow and lies at $9 \mathrm{MPa}$. We attribute this increase to a sensitivity of our state equations to the liquid-solid phase transition, which is affected by the presence of ions ${ }^{26}$. A similar behaviour was observed for electrons ${ }^{36}$. The decrease of the radii with increasing pressures is attributed to compression.

For known particle densities inside spherical snowballs it is possible to associate the radius with the content of helium atoms in the cluster. The particle densities $n$ are not known but can be approximated between a higher limit of densities reported for solid helium $\left(0.03039 \AA^{-3}\right.$ at $2.18 \mathrm{~K}$, $2.5 \mathrm{MPa}$ ) and a lower limit of densities reported for liquid helium $\left(0.02194 \AA^{-3}\right.$ at $\left.2.18 \mathrm{~K}, 0.006 \mathrm{MPa}\right)$. Along the saturated vapour pressure line the radius, $r$, varies from $0.53 \mathrm{~nm}$ to $0.68 \mathrm{~nm}$. Using $N=n V=n \frac{4}{3} \pi r^{3}$ we obtain $N=14$ and 19 atoms for $\mathrm{r}=0.53 \mathrm{~nm}$ and $N=29$ and 40 atoms for $\mathrm{r}=0.68 \mathrm{~nm}$ in the lower and higher number density limit, respectively.

For all temperatures the agreement between theoretical mobilities and radii derived through Equation (3) and experimental data is very good. We note that in the low pressure region at $2.2 \mathrm{~K}$ the experimental data is scattered, making it difficult to deduce the compressibility of the snowball-in-helium system. Summarising, the evolution of hydrodynamic radius with pressure can be interpreted that even at the lowest pressures of liquid helium ion-clusters are fully developed. 


\subsection{Interaction of positive snowballs}

Equations (2) and (3) represent a state equation for positive ions in normal liquid helium in the limit of small concentrations of ions. For such small concentrations the state equation is representative for interactions between ions and their nearest neighbours which are neutral helium atoms. Furthermore, the state equation represents the interaction of the entire ion cluster with helium atoms.

The good fit of a van der Waals type state equation characterised by a $\Pi$ term proportional to $\alpha \rho^{2}$ is remarkable in several ways. The non-covalent interaction energy between an ion and a neutral, polarisable atom or molecule should be governed by a $r^{-4}$ dependence on their separation, $r$. The proportionality of the $\Pi$ term to $\alpha \rho^{2}$ shows that $r^{-6}$ scaling is dominant in the interaction between an ion cluster and helium atoms and that electrostriction, while being the driving force, is not visible in the interaction potential of an ion cluster and the neutral helium atoms within the liquid phase.

The value for $\alpha=0.00796$ bar $\mathrm{m}^{6} / \mathrm{kg}^{2}$ found for the van der Waals equation for positive ion clusters in normal liquid helium is 5.7 times larger than that of pure helium $\left(\alpha_{\text {pureHe }}=\right.$ 0.0014 bar $\mathrm{m}^{6} / \mathrm{kg}^{2}$ as derived from critical parameters), reflecting the solvation of positive ion clusters in helium.

Furthermore we note that a predominant proportionality of $\Pi$ to $\alpha \rho^{2}$ as expressed through (5) was not observed for electrons in normal liquid helium ${ }^{36}$, suggesting a sharper interface of positive ion clusters than for clusters forming around solvated electrons in helium.

\subsection{Structure of the Atkins' snowballs}

The notion of a solid snowball surrounding a positive ion embedded in liquid helium is very well established ${ }^{11,17,19,22,23}$. It has been suggested that increased pressure may cause structural transitions ${ }^{46}$. To test this hypothesis we will analyse the pressure dependence of the simulated hydrodynamic radii and calculate the compressibilities of the snowballs as a function of hydrostatic pressure. Inspection of the compressibility shows that snowballs also exhibit liquid-like behaviour.

The isothermal compressibility $\beta$ is defined as

$$
\beta=-\frac{1}{V}\left(\frac{\partial V}{\partial P}\right)_{T}
$$

Assuming spherical snowballs the compressibility can be readily derived from the derivative of the hydrodynamic radius. Compressibilities derived from the steeply decreasing radius show that for lower pressures the ion-clusters behave liquid-like.

Compressibilities of liquid and solid helium are shown in Table 1. The compressibilities of $\mathrm{He}_{n}^{+}$clusters in liquid helium
Table 1 Representative values of $\beta$ for the liquid and solid phases of helium as reported in ${ }^{47,48}$. The value at $0 \mathrm{~K}$ has been interpolated from data; the mean $\beta$ at $1.1 \mathrm{~K}$ was measured between the solidus and $66 \mathrm{~atm}$

\begin{tabular}{lll} 
Phase & $\beta\left(\mathrm{Pa}^{-1}\right)$ & $(T, P)$ \\
\hline liquid & $1.16 \times 10^{-7}$ & $3 \mathrm{~K}, 4.5 \mathrm{~atm}$ \\
liquid & $3.80 \times 10^{-6}$ & $5 \mathrm{~K}, 2 \mathrm{~atm}$ \\
solid & $1.7 \times 10^{-8}$ & $0 \mathrm{~K}, 66 \mathrm{~atm}$ \\
solid & $3.1 \times 10^{-8}$ & mean $\beta$ at $1.1 \mathrm{~K}$ \\
\hline
\end{tabular}

are in the same order of magnitude as the compressibilities reported for liquid helium at 3-5 $\mathrm{K}^{47}$ and superfluid helium ${ }^{49,50}$, and are far higher than those of solid ${ }^{4} \mathrm{He}^{48,51}$ :

Fig. 4 shows a comparison of the compressibility of snowballs in liquid helium for two different temperatures. We have also indicated the lowest compressibility observed for liquid helium and the highest compressibility observed for solid helium. We can observe that for several thermodynamic states the compressibility of the snowball lies above the lowest reported of the liquid phase, and for a few others it lies below, indicating pressure regions where the snowballs are liquid-like or solid-like, if the compressibility is taken as a criterion of solidity/liquidity of the snowball. The region on the $4.2 \mathrm{~K}$ isotherm between the lowest $\beta$ in the liquid and the highest $\beta$ in the solid can be taken as the fusion region at this temperature and the error for this assignment is given by the range of pressures spanned by $\beta(P)$ in the transition region.

Contrasting the curves for 2.63 and $4.2 \mathrm{~K}$ suggests that the phase transition between solid and liquid-like phase depends on the temperature. Higher temperatures facilitate melting. Melting is also facilitated by the rather small size of the clusters. Clusters exhibit lower melting temperatures than their bulk counterparts ${ }^{52}$.

Hence, the calculated compressibilities support the idea of liquidity of the $\mathrm{He}_{n}^{+}$clusters or at least of parts of the cluster $^{53}$. A liquid cluster or even a cluster with a liquid surface would allow the helium atoms to change their role as solvent or solute particles. Such dynamical effects are expected in hydrated ions.

\section{Conclusions}

The pressure dependence of positive ion mobility in normal liquid helium has been modelled for several isotherms by adopting a thermodynamic approach. A van der Waalstype state equation, exhibiting proportionality between internal pressure, $\Pi$ and the density $\rho$, was found to describe the variation with pressure in the best possible way. This behaviour is different from what we found earlier for electrons in normal liquid helium and suggests a sharp interface between cluster and fluid. By adopting the free-volume model the hy- 


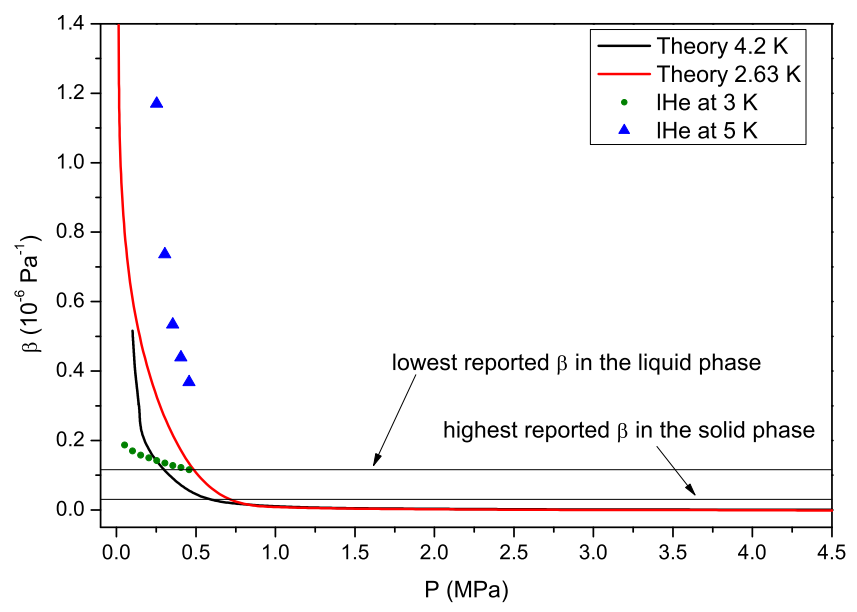

Fig. 4 Isothermal compressibility of snowballs $\beta$ as a function of pressure for the isotherms at $4.2 \mathrm{~K}$ and $2.63 \mathrm{~K}$ compared with that of liquid and solid helium. The lowest measured $\beta$ in the liquid phase and the highest measured $\beta$ in the solid phase from Table 1 are indicated. A phase transition in the intermediate region takes place from the liquid to the solid phase of the snowball.

drodynamic radius of the positive ions were calculated giving an account of the size of the snowball-clusters and their mobility, which was found in very good agreement with experiment. This knowledge enabled us to calculate the isothermal compressibility $\beta$ of the snowballs as a function of pressure. Inspection of $\beta$ revealed liquid-like features for low hydrodynamic pressures up to about $0.5 \mathrm{MPa}$. The liquid-like state of ion clusters, contradicting their common designation as 'snowballs', was attributed to their elevated temperature of 2 to $4 \mathrm{~K}$ and the fact that clusters show lower melting temperatures than their bulk counterparts.

The excellent agreement between the calculated and experimentally determined mobilities suggests that the thermodynamic state equation found for the snowball-helium mixtures is valid and that mobility measurements can be used to validate thermodynamic state equations of other mixtures of ions and solvents. Conversely, mobility data can be predicted for conditions that are experimentally not accessible. We expect that a similar form of $\Pi$ is suitable for similar snowball-like systems, most notably those composed of foreign ions within a cluster of helium and encourage measurements of their mobility to test this hypothesis.

Furthermore, we expect state equations describing snowball-like systems composed of other solvents, such as the heavier rare gases, i.e. $\mathrm{Ne}^{+} \mathrm{Ne}_{n}, \mathrm{Ar}^{+} \mathrm{Ar}_{n}$ etc. to appear similar to liquid helium. Ultimately, our method has potential to be extended to these and other systems.

\section{Acknowledgements}

The authors kindly acknowledge critical reading of the manuscript and discussions with Jussi Eloranta, Northridge and Manuel Barranco, Barcelona. K. von Haeften and F. Aitken acknowledge funding by the British Council through the Alliance Programme. K. von Haeften is grateful for financial support through The Leverhulme Trust (Research Grant F00212AH), the Royal Society (International Exchange Grant RP16G0679) and the Université Joseph Fourier for a Visiting Professorship. Luis Guillermo Mendoza Luna acknowledges financial support from the Mexican Consejo Nacional de Ciencia y Tecnología (CONACYT) Scholarship number 310668, ID 215334.

\section{References}

1 K. Wamba, C. Hall, M. Breidenbach, R. Conley, A. Odian, C. Prescott, P. Rowson, J. Sevilla, K. Skarpaas, R. DeVoe et al., Nucl. Instr. Meth. Phys. Res. A, 2005, 555, 205-210.

2 D. J. Tobias and J. C. Hemminger, Science, 2008, 319, 1197-1198.

3 R. Shi and Y. Wang, J. Phys. Chem. B, 2013, 117, 5102-5112.

4 K. Fujii, H. Hamano, H. Doi, X. Song, S. Tsuzuki, K. Hayamizu, S. Seki, Y. Kameda, K. Dokko, M. Watanabe et al., J. Phys. Chem. C, 2013, 117, 19314-19324.

5 G. Ren, R. Shi and Y. Wang, J. Phys, Chem. B, 2014, 118, 4404-4411.

6 M. Tam and H. H. Hill, Analyst, 2011, 136, 3098-3106.

7 M. R. Lamabadusuriya, W. F. Siems, H. H. Hill Jr, A. Mariano and S. K. Guharay, Anal. Chem., 2012, 84, 9295-9302.

8 J. Hubbard, R. Kayser and P. Stiles, Chem. Phys. Lett., 1983, 95, 399 401.

9 S. Jeng, W. Fairbank Jr and M. Miyajima, J. Phys. D: Appl. Phys., 2009, 42, 035302.

10 L. Meyer and F. Reif, Phys. Rev., 1958, 110, 279.

11 K. Atkins, Phys. Rev., 1959, 116, 1339.

12 L. Meyer and F. Reif, Phys. Rev., 1961, 123, 727.

13 M. W. Cole and R. A. Bachman, Phys. Rev. B, 1977, 15, 1388.

14 M. Ovchinnikov, B. Grigorenko, K. Janda and V. Apkarian, J. Chem. Phys., 1998, 108, 9351.

15 D. Mateo and J. Eloranta, J. Phys. Chem. A, 2014, 118, 6407-6415.

16 W. I. Glaberson and W. W. Johnson, J. Low Temp. Phys., 1975, 20, 313338.

17 N. Takahashi, T. Shimoda, Y. Fujita, T. Itahashi and H. Miyatake, Z Phys $B, 1995,98,347-351$.

18 S. Müller, M. Mudrich and F. Stienkemeier, J. Chem. Phys., 2009, 131, 044319.

19 P. Bartl, C. Leidlmair, S. Denifl, P. Scheier and O. Echt, J. Phys. Chem. A, 2013, 118, 8050-8059.

20 M. Buzzacchi, D. Galli and L. Reatto, Phys. Rev. B, 2001, 64, 094512.

21 M. Rossi, M. Verona, D. Galli and L. Reatto, Phys. Rev. B, 2004, 69, 212510.

22 E. Coccia, E. Bodo and F. A. Gianturco, Eur. Phys. Lett., 2008, 82, 23001.

23 P. Slavicek and M. Lewerenz, Phys. Chem. Chem. Phys., 2010, 12, $1152-$ 1161.

24 A. Leal, D. Mateo, A. Hernando, M. Pi, M. Barranco, A. Ponti, F. Cargnoni and M. Drabbels, Phys. Rev. B, 2014, 90, 224518.

25 K. W. Schwarz, Adv. Chem. Phys., 1975, 33, 3.

26 P. Moroshkin, A. Hofer, S. Ulzega and A. Weis, Nature Phys., 2007, 3, 786-789. 
27 T. Döppner, T. Diederich, J. Tiggesbäumker and K.-H. Meiwes-Broer, Eur. Phys. J. D, 2001, 16, 13-16.

28 T. Döppner, T. Diederich, S. Göde, A. Przystawik, J. Tiggesbäumker and K.-H. Meiwes-Broer, J. Chem. Phys., 2007, 126, 244513.

29 M. Theisen, F. Lackner and W. E. Ernst, Phys. Chem. Chem. Phys., 2010, 12, 14861-14863.

30 F. Grandinetti, Int. J. Mass Spectrom., 2004, 237, 243-267.

31 S. L. Fiedler, D. Mateo, T. Aleksanyan and J. Eloranta, Phys. Rev. B, 2012, 86, 144522.

32 H. T. Davis, S. A. Rice and L. Meyer, Phys. Rev. Lett., 1962, 9, 81-83.

33 K. Keshishev, Y. Kovdrya, L. Mezhov-Deglin and A. Shalnikov, Sov. Phys. JETP, 1969, 56, 94

34 L. Meyer, H. Davis, S. Rice and R. Donnelly, Phys. Rev., 1962, 126, 1927-1934.

35 K. W. Schwarz, Phys. Rev. A, 1972, 6, 837.

36 F. Aitken, Z. Li, N. Bonifaci, A. Denat and K. von Haeften, Phys. Chem. Chem. Phys., 2011, 13, 719-724.

37 F. Aitken, N. Bonifaci, A. Denat and K. von Haeften, J. Low Temp. Phys., 2011, 162,1-8.

38 A. Doolittle, J. Appl. Phys., 1951, 22, 1471.

39 M. Cohen and D. Turnbull, J. Chem. Phys., 1959, 31, 1164.

40 T. Miyamoto and K. Shibayama, J. Appl. Phys., 1973, 44, 5372.

41 N. Gee and G. R. Freeman, Can. J. Chem., 1989, 67, 27-31.

42 N. Gee and G. R. Freeman, J. Chem. Phys., 1989, 90, 5399-5405.

43 M. Kuchnir, Ph.D. thesis, University of Illinois, 1966.

44 P. J. Linstrom and W. G. Mallard, NIST Chem. webbook; http://webbook.nist.gov/chemistry/ [webbook.nist.gov]. NIST standard reference database No. 69, 2001.

45 F. Aitken, N. Bonifaci, A. Denat and K. von Haeften, IEEE Int. Conf. Conduction and Breakdown (ICDL), 2011, pp. 1-4.

46 M. W. Cole and F. Toigo, Phys. Rev. B, 1978, 17, 2054.

47 M. H. Edwards and W. C. Woodbury, Can. J. Phys., 1961, 39, 1833-1841.

48 D. Henshaw, Phys. Rev., 1958, 109, 328.

49 E. Grilly, Phys Rev, 1966, 149, 97.

50 J. S. Brooks and R. J. Donnelly, J. Phys. Chem. Ref. Data, 1977, 6, 51104.

51 C. P. Herrero, J Phys: Cond. Mat., 2008, 20, 295230.

52 W. H. Qi and M. P. Wang, J. Mater. Sci. Lett., 2002, 21, 1743-1745.

53 K. von Haeften, T. Laarmann, H. Wabnitz and T. Möller, J. Electron. Spectrosc. Relat. Phenom., 2000, 106, 199-206. 